\title{
The Application of the Boundary Element Method to the Problem of Wave Diffraction from a Diamond Shaped Inclusion
}

\author{
Yasuhide Shindo*, Tasuku Nakamura and Fumio Narita
}

Department of Materials Processing, Graduate School of Engineering, Tohoku University, Aoba-yama 6-6-02, Sendai 980-8579, Japan

\begin{abstract}
We investigate the scattering of in-plane compressional (P) and shear (SV) waves by diamond shaped inclusions using a boundary element method. Special case that the shape of the diamond becomes square is also considered. Numerical results for some diamond shapes of the inclusion are obtained, and the effects of frequency on the scattering cross sections are discussed in detail. The response simplifies for the limited cases of a hole and a rigid inclusion, but with quite different behavior for each. Calculations for a diamond shaped SiC-fiber-reinforced Al composite are also carried out, and the results are shown graphically.
\end{abstract}

\section{INTRODUCTION}

Elasticity solutions involving the static and dynamic behavior of inclusions embedded in dissimilar materials have received a good deal of attention, partly because of the growing applications for composites. The problem of a single polygonal inclusion in an elastic matrix has been examined by several authors. Chen [1] studied the singular stress fields created by an antiplane deformation at a diamond inclusion corner. Reedy and Guess [2] considered a rigid square inclusion embedded within an elastic disk, and discussed the stress state generated by the inclusion. Recently, Pan and Jiang [3] investigated the stress singularity at vertices of regular polygonal inclusions with various numbers of sides, and revealed the effects of material orientation and corner angle at the vertex on the elastic singularity.

The evaluation of elastic waves propagating in composites is fundamental to the investigation of microstructure. This plays a significant role in design, development, processing and in-service inspection of the composites. The scattering of elastic waves by inclusions has been studied by several authors $[4,5]$.

In this paper, we study the effect of inclusion shape on the scattering of time-harmonic in-plane compressional (P) and shear (SV) waves by a diamond shaped inclusions. Under the plane strain assumption, the solutions are obtained using the boundary element method. Numerical results are given as a function of frequency, and the effect of cross sectional shape of the diamond shaped inclusion on the scattering cross sections are discussed in detail. Numerical calculations are also examined for a diamond shaped SiC-fiberreinforced Al composite.

*Address correspondence to this author at the Department of Materials Processing, Graduate School of Engineering, Tohoku University, Aobayama 6-6-02, Sendai 980-8579, Japan; Tel/Fax: +81-22-795-7341; E-mail: shindo@material.tohoku.ac.jp

\section{PROBLEM STATEMENT AND SCATTERED FAR FIELD}

Consider a diamond shaped inclusion inscribed into a circle of radius $a$ embedded in an infinite matrix. The Cartesian coordinate system $\left(x_{1}, x_{2}, x_{3}\right)$ with origin at center of the circle will be used. Let $\lambda, \mu, \rho, v$ be the Lamé constants, mass density, Poisson's ratio of the matrix, and $\lambda_{0}, \mu_{0}, \rho_{0}, v_{0}$ those of the inclusions. The geometry is depicted in Fig. (1), where $\delta$ is the angle defining the orientation of inclusions, $\theta$ is the corner angle, $\Gamma$ is the surface of inclusions, and $D$ and $C$ are the domains outside and inside inclusions, respectively.

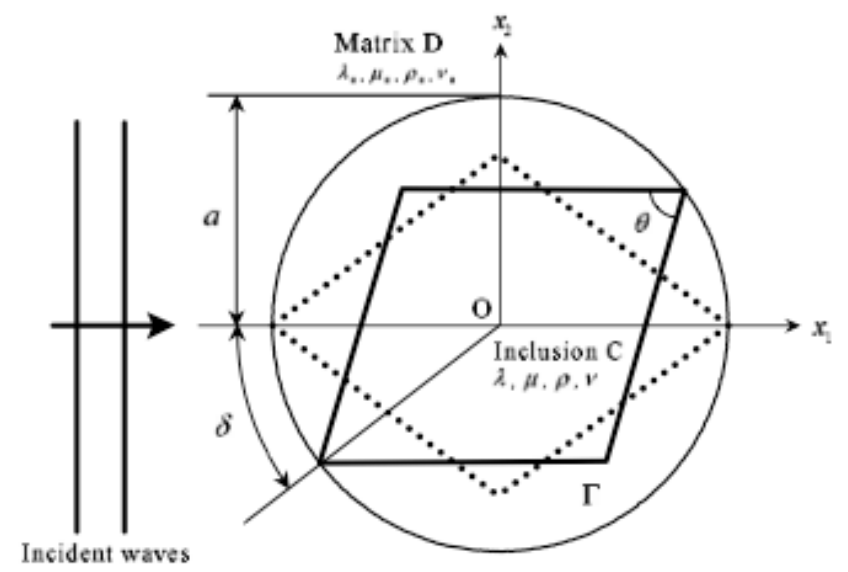

Fig. (1). Diamond shaped inclusion and coordinate systems.

The components in the $x_{1}$ - and $x_{2}$-directions of the displacement vector $\mathbf{u}$ are $u_{1}$ and $u_{2}$, while the component $u_{3}$ is absent because the problem is plane strain. For the same reason, derivatives with respect to $x_{3}$ are zero. The time factor $\exp (-i \omega t)$, which is common to all field vari- 
ables in a steady-state regime, is omitted throughout this paper; $\omega$ is the circular frequency and $t$ is the time. The stress equations of motion are given by

$\sigma_{j i, j}+\rho \omega^{2} u_{i}=0$

where a comma followed by an index denotes partial differentiation with respect to the space coordinate $x_{i}$, indices can assume the values 1 and 2 only, and $\sigma_{i j}$ define the components of stress tensor. We have introduced the summation convention for repeated tensor indices. The stress components are related to the displacement gradients by Hooke's law

$\sigma_{i j}=\lambda \delta_{i j} u_{k, k}+\mu\left(u_{i, j}+u_{j, i}\right)$

$\sigma_{33}=\lambda u_{k, k}$

where $\delta_{i j}$ is the Kronecker delta.

Let us consider a plane compressional $(\mathrm{P})$ wave propagation in the positive $x_{1}$-direction or a plane shear (SV) wave polarized in the $x_{2}$-direction and propagating in the positive $x_{1}$-direction. The appropriate displacement vector of the equation of motion can be expressed as

$\mathrm{u}^{i}(\mathrm{x})=u_{0} \exp \left(i k_{p} x_{1}\right) \mathrm{e}_{1}+w_{0} \exp \left(i k_{s v} x_{1}\right) \mathrm{e}_{2}$

where the superscript $i$ stands for the incident component, $\mathbf{x}$ $=x_{1} e_{1}+x_{2} e_{2}$ denotes the position vector, $\mathbf{e}_{1}$ and $\mathbf{e}_{2}$ are the unit base vectors in the $x_{1}$ - and $x_{2}$-directions, $u_{0}$ and $w_{0}$ are the amplitudes of the incident $\mathrm{P}$ and $\mathrm{SV}$ waves, $k_{p}=\omega / c_{p}$ and $k_{s v}=\omega / c_{s v}$ are the wave numbers of the $\mathrm{P}$ and SV waves in the matrix, and $c_{p}=\{(\lambda+2 \mu) / \rho\}^{1 / 2}$ and $c_{s v}=(\mu / \rho)^{1 / 2}$ are the $\mathrm{P}$ and SV wave speeds in the matrix. The complete solutions, $u_{i}, \sigma_{i j}$, are given by

$u_{i}=u_{i}^{i}+u_{i}^{s}, \sigma_{i j}=\sigma_{i j}^{i}+\sigma_{i j}^{s}$

where the superscript $s$ denotes the scattered component within the matrix. In addition, the scattered field must fulfill the radiation condition.

The boundary conditions for the scattered field are shown as

$u_{i}=u_{i}^{t}, t_{i}+t_{i}^{t}=0$

where the superscript $t$ stands for the transmitted component within an inclusion, $t_{i}=\sigma_{j i} n_{j}$ and $t_{i}^{t}=\sigma_{j i}^{t} n_{j}^{t}$ are the components of the traction vectors $\mathrm{t}$ and $\mathrm{t}^{t}$ exerted by the inclusion on the matrix and by the matrix on the inclusion, and $n_{j}$ and $n_{j}^{t}$ are the components of the unit outward normal vectors $\mathrm{n}$ from $D$ and $\mathrm{n}^{t}$ from $C$, respectively. The boundary integral equation for the matrix is

$$
\begin{aligned}
& c_{i j}(\mathrm{x}) u_{j}(\mathrm{x})=\int_{\Gamma} U_{i j}(\mathrm{x}, \mathrm{y}) t_{j}(\mathrm{y}) d \Gamma(\mathrm{y}) \\
& -\int_{\Gamma} T_{i j}(\mathrm{x}, \mathrm{y}) u_{j}(\mathrm{y}) d \Gamma(\mathrm{y})+u_{i}^{i}(\mathrm{x})
\end{aligned}
$$

where $\mathrm{x}=x_{1} \mathrm{e}_{1}+x_{2} \mathrm{e}_{2}$ and $\mathrm{y}=y_{1} \mathrm{e}_{1}+y_{2} \mathrm{e}_{2}$ are the field and source points, respectively, and the coefficients of $c_{i j}$ depend on the local geometry of $\Gamma$ at $\mathrm{x} \cdot U_{i j}(\mathrm{x}, \mathrm{y})$ and $T_{i j}(\mathrm{x}, \mathrm{y})$ are the fundamental solutions for displacement and traction, and are given in Appendix A. The boundary integral equation for the inclusion is

$$
\begin{aligned}
& c_{i j}^{t}(\mathrm{x}) u_{j}^{t}(\mathrm{x})=\int_{\Gamma} U_{i j}^{t}(\mathrm{x}, \mathrm{y}) t_{j}^{t}(\mathrm{y}) d \Gamma(\mathrm{y}) \\
& -\int_{\Gamma} T_{i j}^{t}(\mathrm{x}, \mathrm{y}) u_{j}^{t}(\mathrm{y}) d \Gamma(\mathrm{y})
\end{aligned}
$$

where the coefficients of $c_{i j}^{t}$ depend on the local geometry of $\Gamma$ at $\mathrm{x}, U_{i j}^{t}$ and $T_{i j}^{t}$ are also defined by Eqs. (A.1) and (A.2), but with the $\mathrm{P}$ and $\mathrm{SV}$ wave speeds $c_{p}^{t}=\left\{\left(\lambda_{0}+2 \mu_{0}\right) / \rho_{0}\right\}^{1 / 2}$ and $c_{s v}^{t}=\left(\mu_{0} / \rho_{0}\right)^{1 / 2}$ of the inclusion, and the unit outward normal vector $\mathrm{n}^{t}$.

Equations (7) and (8) can be written for each node. After the discretization of the boundary into quadratic elements, we write Eq. (7) for the matrix as

$\mathrm{Hu}=\mathrm{Gt}+\mathrm{u}^{i}$,

where the matrices $\mathrm{H}$ and $\mathrm{G}$ contain the integrals of the traction and displacement kernels, respectively, and $\mathrm{u}^{i}$ is the incident wave which impinges on the inclusion. For the inclusion, the boundary integral equation (8) becomes

$\mathrm{H}^{t} \mathrm{u}^{t}=\mathrm{G}^{t} \mathrm{t}^{t}$.

Taking the inverse of the matrix $G\left(G^{t}\right)$ in Eq. (9) (in Eq. (10)) and operating with the inverse matrix on both sides of Eq. (9) (of Eq. (10)), we obtain

$$
\begin{aligned}
& \mathrm{Ku}=\mathrm{t}+\mathrm{f}^{i}, \\
& \mathrm{~K}^{t} \mathrm{u}^{t}=\mathrm{t}^{t},
\end{aligned}
$$

where

$\mathrm{K}=\mathrm{G}^{-1} \mathrm{H}$,

$\mathrm{K}^{t}=\left(\mathrm{G}^{t}\right)^{-1} \mathrm{H}^{t}$,

$\mathrm{f}^{i}=\mathrm{G}^{-1} \mathrm{u}^{i}$.

Addition of Eqs. (11) and (12) yields with the help of the equation for the boundary condition (6) that

$\left(\mathrm{K}+\mathrm{K}^{t}\right) \mathrm{u}=\mathrm{f}^{i}$.

From this equation, we can compute the displacements on the surface of the inclusion.

For $|\mathrm{x}| \rightarrow \infty$, the scattered fields can be represented by 


$$
\begin{array}{r}
u_{i}^{s}(\mathbf{x}) \sim u_{0} A_{i}(\theta, \delta) \sqrt{\frac{2}{\pi k_{p}|\mathbf{x}|}} \exp \left\{i\left(k_{p}|\mathbf{x}|-\frac{\pi}{4}\right)\right\} \\
+w_{0} B_{i}(\theta, \delta) \sqrt{\frac{2}{\pi k_{s v}|\mathbf{x}|}} \exp \left\{i\left(k_{s v}|\mathbf{x}|-\frac{\pi}{4}\right)\right\}
\end{array}
$$

where $\theta$ is the scattering angle measured from $x_{1}$-axis, and the terms $A_{i}$ and $B_{i}$ are given in Appendix B. The scattering cross sections for the incident $\mathrm{P}$ and $\mathrm{SV}$ waves are then

$\Sigma_{p}=-\frac{4}{k_{p}} \operatorname{Re}\left[A_{1}(0, \delta)\right]$

$\Sigma_{s v}=-\frac{4}{k_{s v}} \operatorname{Re}\left[B_{2}(0, \delta)\right]$.

\section{NUMERICAL RESULTS AND DISCUSSION}

To investigate the effect of inclusion shape and orientation on the scattering cross section, the far-field scattering amplitudes have been numerically computed for a diamond shaped hole and a diamond shaped rigid inclusion. Diamond shaped SiC-Al composites are also considered. The material properties of $\mathrm{SiC}$ and $\mathrm{Al}$ are listed in Table 1. Special treatment is given to the case where a sharp corner of the diamond shaped inclusion occurs on an interface boundary between regions. The idea is that the structure with sharp corners should be approximated by a structure with rounded corners [4].

Table 1. Material Properties of $\mathrm{SiC}$ and $\mathrm{Al}$

\begin{tabular}{|c|c|c|c|c|}
\hline & $\rho\left(\mathrm{kg} / \mathrm{m}^{3}\right)$ & $\mu(\mathrm{GPa})$ & $\lambda+2 \mu(\mathrm{GPa})$ & $\nu$ \\
\hline $\mathrm{SiC}$ & 3181 & 188.1 & 474.2 & 0.17 \\
\hline & $\rho_{0}\left(\mathrm{~kg} / \mathrm{m}^{3}\right)$ & $\mu_{0}(\mathrm{GPa})$ & $\lambda_{0}+2 \mu_{0}(\mathrm{GPa})$ & $v_{0}$ \\
\hline $\mathrm{Al}$ & 2706 & 26.7 & 110.5 & 0.34 \\
\hline
\end{tabular}

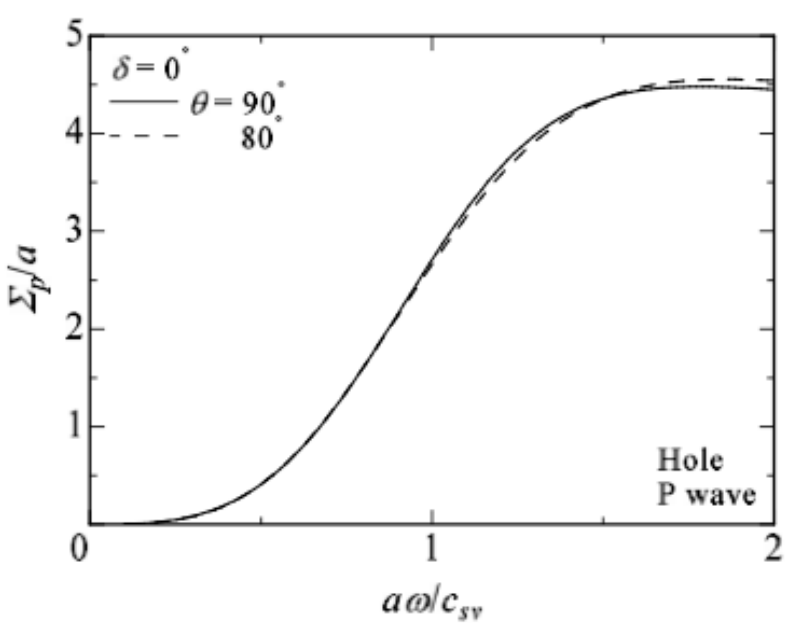

Fig. (2). Scattering cross section versus frequency for square and diamond shaped holes ( $\mathrm{P}$ wave, $\theta=90^{\circ}, 80^{\circ}$ ).

Fig. (2) shows the variation of the scattering cross section $\Sigma_{p} / a$ of the $\mathrm{P}$ wave with the frequency $a \omega / c_{s v}$ for square $\left(\theta=90^{\circ}\right)$ and diamond $\left(\theta=80^{\circ}\right)$ shaped holes at an orientation of angle $\delta=0^{\circ}$. Small difference of the scattering cross section is observed between the corner angles $90^{\circ}$ and $80^{\circ}$. Further decreasing of $\theta$ results in an increase in the scattering cross section (Fig. 3). Similar phenomena are observed for $\delta=45^{\circ}$ and $90^{\circ}$ (not shown). Fig. (4) gives a plot of $\Sigma_{p} / a$ against the orientation angle $\delta$ of diamond shaped hole for a fixed value of $a \omega / c_{s v}=2.0$ while $\theta$ varies from $20^{\circ}$ to $80^{\circ}$. The orientation angle infiuences the scattering cross section.

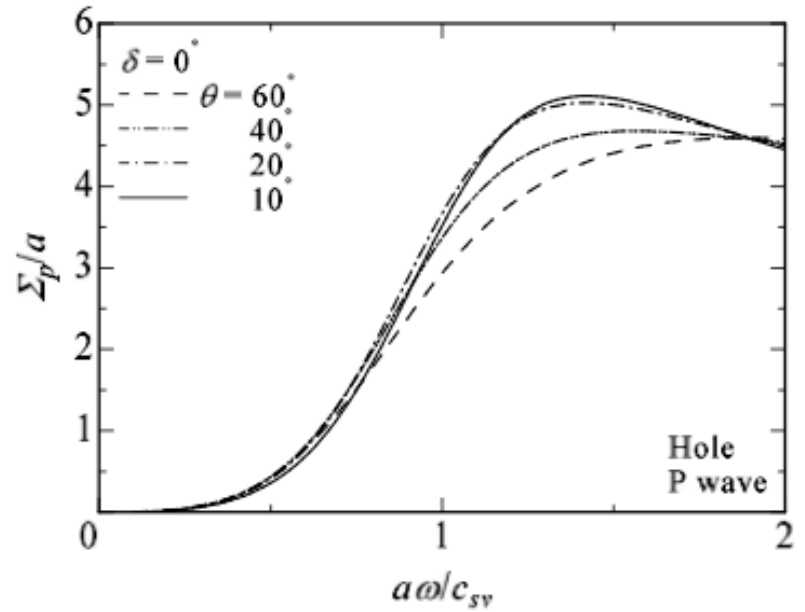

Fig. (3). Scattering cross section versus frequency for diamond shaped holes (P wave, $\theta=60^{\circ}, 40^{\circ}, 20^{\circ}, 10^{\circ}$ ).

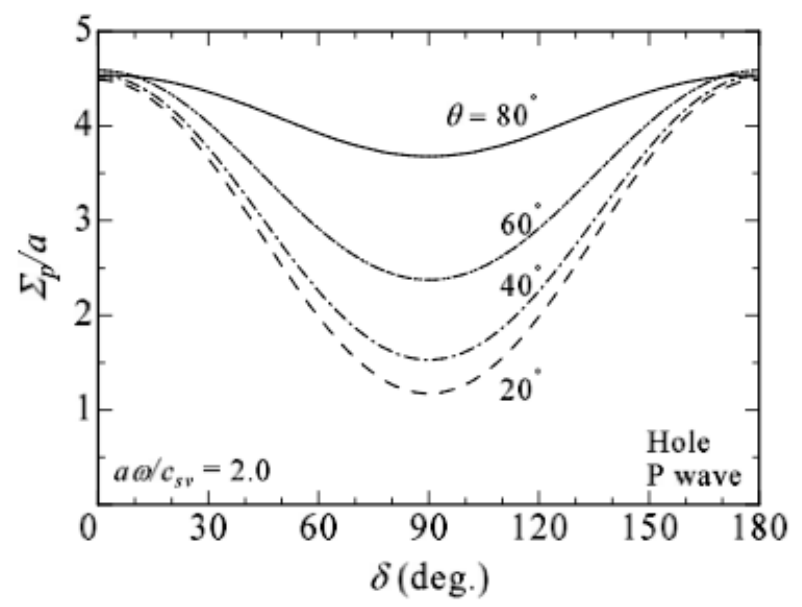

Fig. (4). Scattering cross section versus orientation angle for diamond shaped holes ( $\mathrm{P}$ wave, $a \omega / c_{s v}=2.0$ ).

Fig. (5) shows the variation of $\Sigma_{p} / a$ of the $\mathrm{P}$ wave with $a \omega / c_{s v}$ for square and diamond shaped rigid inclusions at the orientation angle $\delta=0^{\circ}$. The scattering cross section increases with the frequency. The graph also demonstrates the rising $\Sigma_{p} / a$ value with increasing corner angle. Fig. (6) shows $\Sigma_{p} / a$ versus $\delta$ of square and diamond shaped rigid inclusions for $a \omega / c_{s v}=2.0$. The scattering cross section is 
influenced by $\delta$, and peaks at different $\delta$ at large corner angle $\theta$.

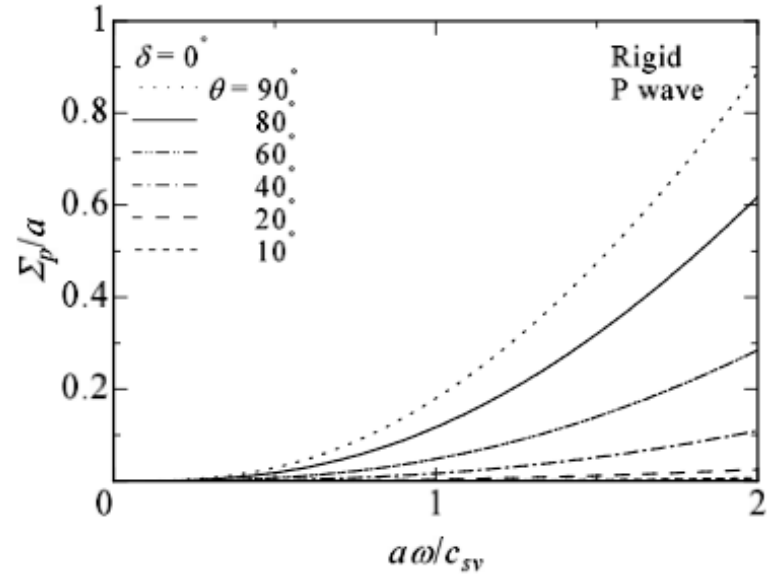

Fig. (5). Scattering cross section versus frequency for square and diamond shaped rigid inclusions ( $\mathrm{P}$ wave).

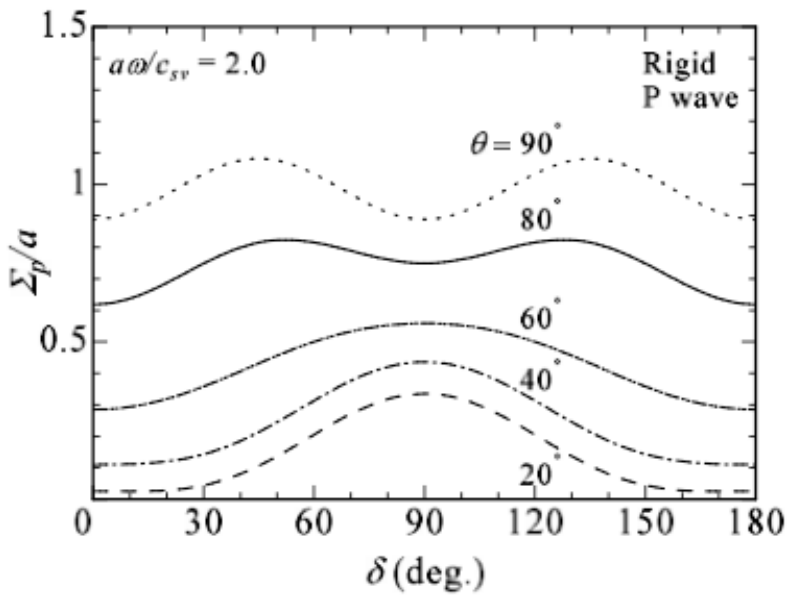

Fig. (6). Scattering cross section versus orientation angle for square and diamond shaped rigid inclusions ( $\mathrm{P}$ wave, $a \omega / c_{s v}=2.0$ ).

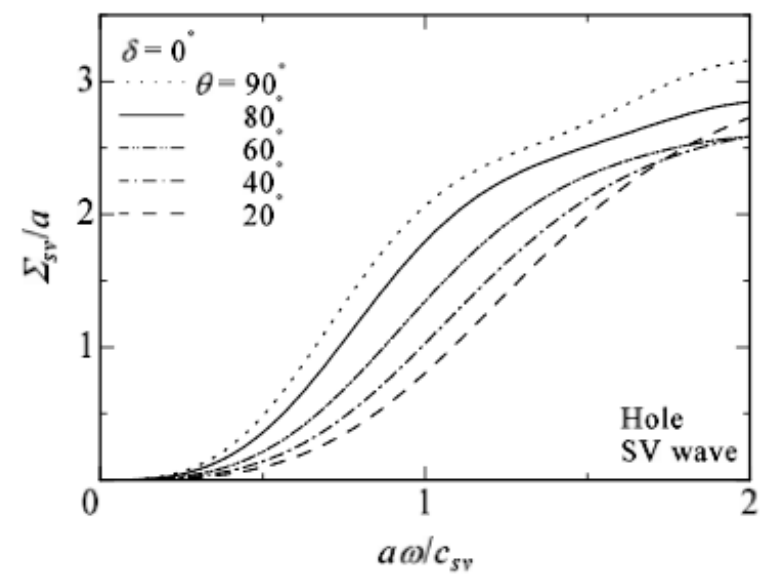

Fig. (7). Scattering cross section versus frequency for square and diamond shaped holes (SV wave).

Fig. (7) exhibits the variation of the scattering cross section $\Sigma_{s v} / a$ of the SV wave with the frequency $a \omega / c_{s v}$ for square and diamond shaped hole with various values of corner angle $\theta$ at the orientation angle $\delta=0^{\circ}$. The scattering cross section of the SV wave decreases with decreasing $\theta$. Fig. (8) also displays the values of $\Sigma_{s v} / a$ of the SV wave with $a \omega / c_{s v}$ for square and diamond shaped rigid inclusions at $\delta=0^{\circ}$. The $\Sigma_{s v} / a$ for rigid inclusions also decreases with decreasing $\theta$. The scattering cross section of the SV wave for both holes and rigid inclusions depends on $\delta$ (not shown).

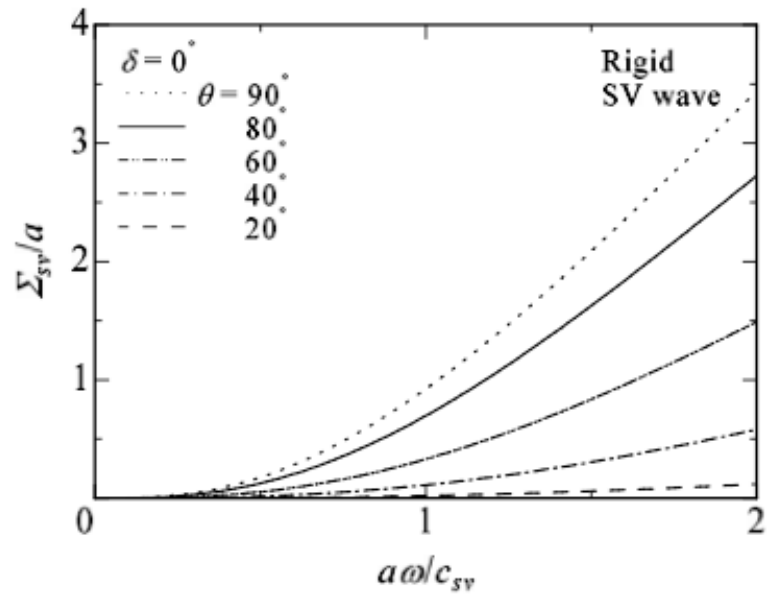

Fig. (8). Scattering cross section versus frequency for square and diamond shaped rigid inclusions ( $\mathrm{SV}$ wave).

The variation of $\Sigma_{p} / a$ of the $\mathrm{P}$ wave with $a \omega / c_{s v}$ for square and diamond shaped $\mathrm{SiC}-\mathrm{Al}$ composites is illustrated in Fig. (9) in which the orientation angle is fixed $\left(\delta=0^{\circ}\right)$. The value of $\Sigma_{p} / a$ of the $\mathrm{P}$ wave for $\mathrm{SiC}-\mathrm{Al}$ composites can be lowered by decreasing $\theta$. Fig. (10) demonstrates the plot of $\Sigma_{p} / a$ of the $\mathrm{P}$ wave against $\delta$ for square and diamond shaped SiC-Al composites. When $\theta$ is large $\left(\theta=90^{\circ}\right.$ and $\left.80^{\circ}\right)$, the $\Sigma_{p} / a$ value has two peaks at $\delta=45^{\circ}$ and $135^{\circ}$. On the other hand, it has only one peak at $\delta=90^{\circ}$ when $\theta$ is small.

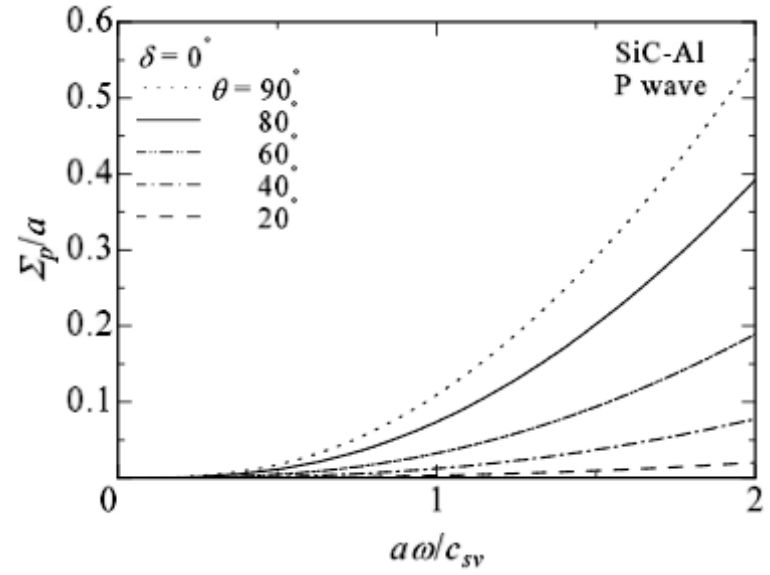

Fig. (9) Scattering cross section versus frequency for square and diamond shaped SiC-Al composites (P wave). 


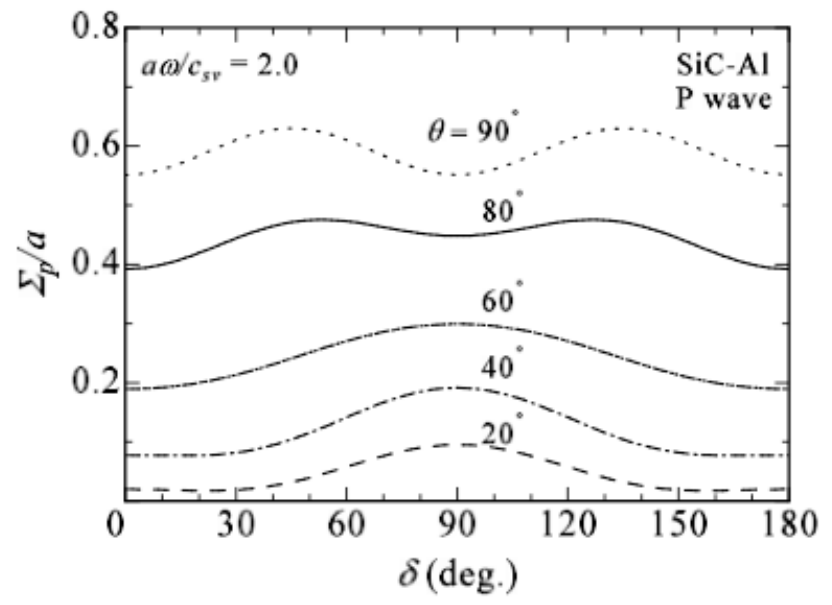

Fig. (10) Scattering cross section versus orientation angle for square and diamond shaped SiC-Al composites (P wave).

\section{CONCLUSIONS}

The scattering of $\mathrm{P}$ and SV waves by diamond shaped inclusions was analyzed using a boundary element method. The numerical solutions were obtained for various finite frequencies, and detailed discussions were given for holes, rigid inclusions and $\mathrm{SiC}-\mathrm{Al}$ composites. It is found that the scattering cross sections are influenced by the corner angle of inclusions. In the case of diamond shaped holes, the scattering cross section strongly depends on the orientation angle. Further work for the modeling of dynamic characteristics of the composites will be carried out.

\section{APPENDIX A}

$U_{i j}(\mathrm{x}, \mathrm{y})$ and $T_{i j}(\mathrm{x}, \mathrm{y})$ in Eq.(7) can be expressed as

$$
\begin{aligned}
& U_{i j}(\mathrm{x}, \mathrm{y})=\frac{1}{2 \pi \mu}\left(\eta \delta_{i j}-\kappa r_{, i} r_{, j}\right), \\
& T_{i j}(\mathrm{x}, \mathrm{y})=\frac{1}{2 \pi}\left\{\left(\frac{d \eta}{d r}-\frac{1}{r} \kappa\right)\left(\delta_{i j} \frac{\partial r}{\partial n}+n_{i} r_{j}\right)\right. \\
& -\frac{2}{r} \kappa\left(n_{j} r_{, i}-2 r_{, i} r_{, j} \frac{\partial r}{\partial n}\right) \\
& \left.-2 \frac{d \kappa}{d r} r_{, i} r_{, j} \frac{\partial r}{\partial n}-\left(2-\frac{c_{p}^{2}}{c_{s v}^{2}}\right)\left(\frac{d \eta}{d r}-\frac{d \kappa}{d r}-\frac{\kappa}{r}\right) r_{, i} n_{j}\right\}
\end{aligned}
$$

where

$\eta=\frac{\pi i}{2} H_{0}\left(\frac{r \omega}{c_{s v}}\right)$

$$
\begin{aligned}
& -\frac{c_{s v}}{i \omega r}\left\{-\frac{\pi}{2} H_{1}\left(\frac{r \omega}{c_{s v}}\right)+\frac{c_{s v}}{c_{p}} \frac{\pi}{2} H_{1}\left(\frac{r \omega}{c_{p}}\right)\right\} \\
& \kappa=-\frac{\pi i}{2} H_{2}\left(\frac{r \omega}{c_{s v}}\right)+\frac{c_{s v}^{2}}{c_{p}^{2}} \frac{\pi i}{2} H_{2}\left(\frac{r \omega}{c_{p}}\right)
\end{aligned}
$$

In the above, $r=|\mathrm{x}-\mathrm{y}|, \partial / \partial n$ denotes the directional differentiation along the unit outward normal vector $\mathrm{n}$ to $\Gamma$, and $H_{l}()$ is the $l$ th order Hankel function of the first kind.

\section{APPENDIX B}

$A_{i}$ and $B_{i}$ in Eq.(17) are

$$
\begin{aligned}
& A_{i}=\frac{1}{u_{0}} \int_{\Gamma}\left\{C_{i j}^{p}(\hat{\mathrm{x}}) t_{j}(\mathrm{y})+k_{p} D_{i j k}^{p}(\hat{\mathrm{x}}) n_{k}(\mathrm{y}) u_{j}(\mathrm{y})\right\} \\
& \times \exp \left(-i k_{p} \hat{\mathrm{x}} \cdot \mathrm{y}\right) d \Gamma(\mathrm{y}), \\
& B_{i}=\frac{1}{w_{0}} \int_{\Gamma}\left\{C_{i j}^{s v}(\hat{\mathrm{x}}) t_{j}(\mathrm{y})+k_{s v} D_{i j k}^{s v}(\hat{\mathrm{x}}) n_{k}(\mathrm{y}) u_{j}(\mathrm{y})\right\} \\
& \times \exp \left(-i k_{s v} \hat{\mathrm{x}} \cdot \mathrm{y}\right) d \Gamma(\mathrm{y})
\end{aligned}
$$

where

$$
\begin{aligned}
& C_{i j}^{p}(\hat{\mathrm{x}})=\frac{i}{4 \rho c_{p}^{2}} \hat{x}_{i} \hat{x}_{j}, \\
& C_{i j}^{s v}(\hat{\mathrm{x}})=\frac{i}{4 \rho c_{s v}^{2}}\left(\delta_{i j}-\hat{x}_{i} \hat{x}_{j}\right), \\
& D_{i j k}^{p}(\hat{\mathrm{x}})=\frac{1}{4} \hat{x}_{i}\left\{\left(1-2 \frac{c_{s v}^{2}}{c_{p}^{2}}\right) \delta_{j k}+2 \frac{c_{s v}^{2}}{c_{p}^{2}} \hat{x}_{j} \hat{x}_{k}\right\}, \\
& D_{i j k}^{s v}(\hat{\mathrm{x}})=\frac{1}{4}\left\{\delta_{i j} \hat{x}_{k}+\delta_{i j} \hat{x}_{j}-2 \hat{x}_{i} \hat{x}_{j} \hat{x}_{k}\right\},
\end{aligned}
$$

and $\hat{\mathrm{x}}=\cos \theta \mathrm{e}_{1}+\sin \theta \mathrm{e}_{2}$ is the unit vector in the $\theta$-direction.

\section{REFERENCES}

[1] Chen DH. Antiplane strain problem of diamond inclusion. Int J Fract 1995; 71: 197-212.

[2] Reedy ED Jr, Guess TR. Rigid square inclusion embedded within an epoxy disk : asymptotic stress analysis. Int J Solids Struct 2001; 38: 1281-93.

[3] Pan E, Jiang X. Singularity analysis at the vertex of polygonal quantum wire inclusions. Mech Res Comm 2006; 33: 1-8.

[4] Sato H, Shindo Y. Scattering of compressional and shear waves by a polygonal inclusion. Mech Adv Mater Struct 2002; 9: 175-87.

[5] Kim JYF. Extinction of elastic wave energy due to scattering in a viscoelastic medium. Int J Solids Struct 2003; 40: 4319-29. 